\title{
Prognostic value and potential function of splicing events in prostate adenocarcinoma
}

\author{
ZHI-GUANG HUANG ${ }^{1,2 *}$, RONG-QUAN HE H. $^{2 *}$ and ZENG-NAN MO ${ }^{1,2}$ \\ ${ }^{1}$ Guangxi Collaborative Innovation Center for Biomedicine, Guangxi Medical University; \\ ${ }^{2}$ Center for Genomic and Personalized Medicine, Guangxi Medical University; \\ ${ }^{3}$ Department of Biochemistry and Molecular Biology, Guangxi Medical University, Nanning, Guangxi 530021, P.R. China
}

Received May 11, 2018; Accepted July 9, 2018

DOI: $10.3892 /$ ijo.2018.4563

\begin{abstract}
Prostate adenocarcinoma (PRAD) is one of the most common types of malignancy in males and at present, effective prognostic indicators are limited. The development of PRAD has been associated with abnormalities in alternative splicing (AS), a requisite biological process of gene expression in eukaryotic cells; however, the prognostic value of AS products and splicing events remains to be elucidated. In the present study, the data of splicing events and the clinical information of PRAD patients were obtained from The Cancer Genome Atlas (TCGA)SpliceSeq and TCGA databases, respectively. A prognostic index (PI) was generated from disease-free survival-associated splicing events (DFS-SEs), which were identified by univariate/multivariate Cox regression analysis. A total of 6,909 DFS-SEs were identified in PRAD. The corresponding genes for the DFS-SEs were significantly enriched in mitochondria and their associated pathways according to Gene Ontology annotation and in the pathways of fatty acid metabolism, oxidative phosphorylation and Huntington's disease according to a Kyoto Encyclopedia of Genes and Genomes pathway analysis. The PI for mutually exclusive exons had the greatest ability to predict the probability of fiveyear disease-free survival of patients with PRAD, with an area under the time-dependent receiver-operating characteristic curve of 0.7606 . Patients with PRAD, when divided into a 'low' and a 'high' group based on their median PI for exon skip values, exhibited a marked difference in disease-free survival (low vs. high, 3,588.45 \pm 250.51 vs. 1,531.08 \pm 136.50 days; $\left.\mathrm{P}=7.43 \times 10^{-9}\right)$. A correlation network between DFS-SEs of splicing factors and non-splicing factors was constructed to determine the potential mechanisms in PRAD, which included the potential regulatory interaction between the splicing event
\end{abstract}

Correspondence to: Professor Zeng-Nan Mo, Center for Genomic and Personalized Medicine, Guangxi Medical University, 22 Shuangyong Road, Nanning, Guangxi 530021, P.R. China

E-mail: zengnanmo@hotmail.com

Key words: prostate adenocarcinoma, splicing event, prognostic index, The Cancer Genome Atlas of splicing factor RNA binding motif protein 5-alternate terminator (AT)-64957 and the splicing event of non-splicing factor heterochromatin protein 1 binding protein 3-AT-939. In conclusion, the PIs derived from DFS-SEs are valuable prognostic factors for patients with PRAD, and the function of splicing events in PRAD deserves further exploration.

\section{Introduction}

Prostate adenocarcinoma (PRAD) is one of the most common malignant tumor types of the male reproductive system and primarily occurs in elderly individuals (aged $>65$ years) $(1,2)$. According to the most recent global statistical data, the number of newly diagnosed PRAD cases in 2012 has reached 1.1 million (3). The morbidity and mortality associated with PRAD are higher in developed than in developing countries. In the US, the predicted number of new cases of PRAD for 2018 is 164,690 and the number of associated mortalities is 29,430 (4). The mortality from PRAD is substantially reduced by early screening for prostate-specific antigen (PSA); However, the number of individuals who die from PRAD is second only to the number of patients that die from lung cancer, and PRAD therefore ranks second among cancer-associated deaths in males (4,5). According to cancer statistics for 2015, 60,300 individuals were diagnosed with PRAD and 26,600 patients succumbed to the disease in China. While these figures are lower than those for the US, the incidence of PRAD is rapidly increasing each year in China (6).

Only a small number of indicators are currently used for predicting the prognosis of PRAD patients, and each indicator has its own advantages and disadvantages. PSA is currently the most commonly used diagnostic screening and prognostic indicator for PRAD; however, its application has poor specificity $(7,8)$. Circulating tumor cells provide excellent prognostic evaluation of PRAD, but their use is limited by high equipment requirements and costs (9). micro (mi)RNAs have also been demonstrated to have a high prognostic value for PRAD, but the technology for the extraction and identification of relevant miRNAs in limited samples remains to be fully developed and implemented $(8,10)$. Therefore, novel and effective predictors for the prognosis of patients with PRAD are urgently required.

One strategy for identifying prognostic predictors for PRAD is the assessment of alternative splicing (AS), but this 
field has remained largely unexplored. AS is an indispensable process in prokaryotic gene expression (11) and is able to turn mRNA precursors into different types of mature mRNAs through different processes to increase the diversity of mRNA types (12). Increasing evidence indicates that AS has an important role in the development of PRAD (13-16). The splice isoforms generated by AS are involved in different aspects of tumor physiology, including growth, apoptosis, infiltration, metastasis, angiogenesis and metabolism (14). The study of AS in tumors is helping to elucidate the mechanisms underlying tumor pathogenicity and may be a strategy in the search for reliable and effective diagnostic and prognostic targets. AS is classified into the following 7 types, based on the type of splicing: Alternate acceptor site (AA), alternate donor site (AD), alternate promoter (AP), alternate terminator (AT), exon skip (ES), mutually exclusive exons (ME) and retained intron (RI). The products of AS are termed splicing events $(17,18)$. In the present study, the percent-spliced-in (PSI) value (ranging from 0 to 1 ) was used to perform an intuitive quantitative analysis and comparison of splicing events $(18,19)$.

AS involves the use of splicing factors as executive proteins, so that changes in splicing factor expression directly cause an abnormal expression of splicing events (20). Numerous studies have confirmed that mutations in splicing factors are closely associated with the development and progression of tumors $(21,22)$ and that the expression levels of splicing factors differ significantly between tumor cells and juxtacancerous tissue $(20,23)$. For instance, serine- and arginine-rich splicing factor 1 was reported to be highly expressed in various tumor types (24), and pre-mRNA processing factor 6 was indicated to promote tumor cell growth (25). Therefore, exploring the potential regulatory association between splicing factors and splicing events may aid in identifying the pathogenic mechanisms underlying tumor development.

In recent years, the analysis of splicing events in tumor research has become possible with the introduction of deep sequencing techniques. The Cancer Genome Atlas (TCGA) database (https://portal.gdc.cancer.gov/) now provides RNA-sequencing data of various types of tumor and juxtacancerous tissues, while no normal tissues were included. Ryan et al (26) used the RNA-seq data provided by TCGA to establish a TCGASpliceSeq database (http://bioinformatics. mdanderson.org/TCGASpliceSeq/index.jsp), which offers a convenient way for researchers to study splicing events in tumors. The aim of the present study was to perform a re-calculation and in-depth analysis to determine the diseasefree survival-associated splicing events (DFS-SEs) in patients with PRAD. The present study endeavored to construct a prognostic index (PI) for DFS-SEs and to evaluate the prognostic values of these indicators in PRAD using time-dependent receiver-operator characteristic (tROC) and Kaplan-Meier curve analyses. In addition, a correlation analysis was used to construct a potential regulatory network to explain the associations between splicing factors and splicing events.

\section{Material and methods}

Data acquirement and organization. The splicing event data for PRAD were downloaded from the TCGASpliceSeq database. In addition, the clinical data for 500 patients with PRAD were acquired from TCGA database.

In total, 91 patients with a 'Person neoplasm cancer status' of 'Discrepancy', 'Not Available' or 'Unknown', 3 patients with a 'patient death reason' of 'Other, non-malignant disease' or 'Unknown' and 9 patients with ' $<30$ ' in 'days to last followup' were excluded, leaving 397 patients. After matching the patients with their TCGASpliceSeq database entries according to their labeling numbers, 394 patients were included in the present study.

'With tumor' in 'person neoplasm cancer status' was regarded as the endpoint. The DFS time in 'days to last followup' was used to calculate the DFS of patients with PRAD.

Identification and analysis of DFS-SEs. Univariate Cox regression analysis was performed to analyze and identify DFS-SEs. Cytoscape software was used to plot the interactive association between the genes corresponding to DFS-SEs. The Database for Annotation, Visualization and Integrated Discovery (DAVID) Functional Annotation Result Summary (https://david.ncifcrf.gov/summary.jsp, version 6.8) was used for Gene Ontology (GO) and Kyoto Encyclopedia of Genes and Genomes (KEGG) pathway enrichment analysis of the genes corresponding to the top 500 most significant DFS-SEs in PRAD. The top five pathways identified by the GO and KEGG analyses were displayed.

Construction of the PI model for PRAD based on splicing events. To construct powerful PIs, multivariate Cox regression analysis was used to exclude irrelevant splicing events. Multivariate Cox regression analyses were performed for the top 10 DFS-SEs that had the highest prognostic values for each splicing type and the top 10 DFS-SEs that had the highest prognostic values for all splicing types. Splicing events with $\mathrm{P}<0.05$ were selected to constitute PIs. The calculation formula was as follows:

$$
P I=\sum_{i}^{n} P S I i * \beta i
$$

where $\beta$ is the regression coefficient.

Evaluation of the prognostic value of PIs. The efficacy of the PIs in predicting the cancer status after five years was evaluated by tROC analysis with the R package 'survivalROC' (27). Kaplan-Meier curve analysis was performed for the DFS time and cancer status of the patients with PRAD to evaluate the prognostic efficacy of the most significant top 10 DFS-SEs and PIs. Univariate Cox regression analysis was used to calculate the hazard ratio (HR) values of the PIs and other clinical parameters for PRAD recurrence.

Construction of a correlation network of DFS-SEs in PRAD. Information on the splicing factors was downloaded from the SpliceAid2 database (http://www.introni.it/splicing.html) (28) and included 66 splicing factors. All splicing events for the splicing factors were identified based on the results of the univariate Cox regression analysis. A total of 22 splicing events with $\mathrm{P}<0.01$ were selected. In addition, the top 50 splicing events with the highest prognostic value according to the univariate Cox analysis were assessed, and events with 


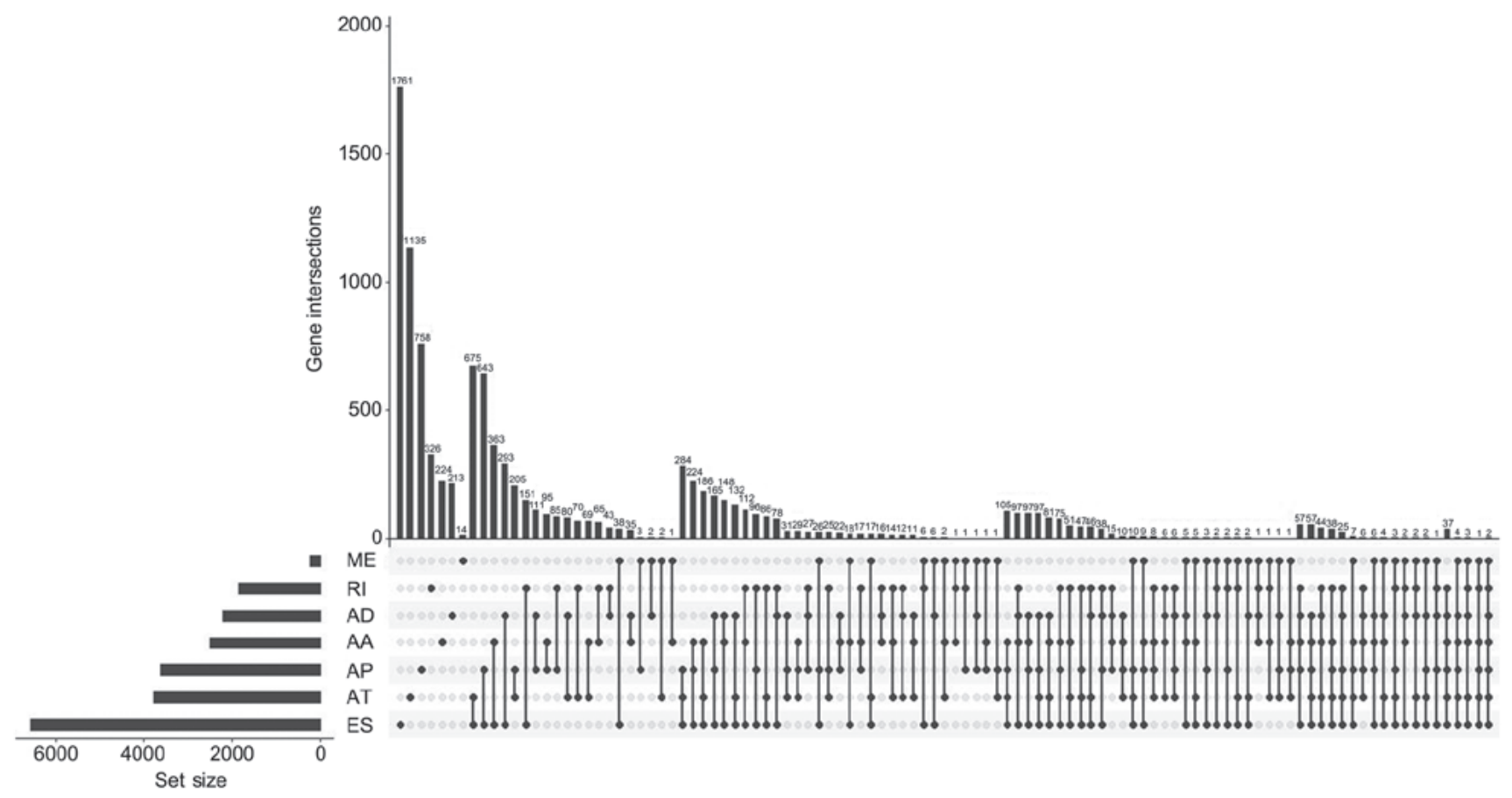

Figure 1. UpSet plots of splicing events in prostate adenocarcinoma. The horizontal axis represents the gene number of seven splicing types. The vertical axis represents the number of genes for one or several splicing types. AA, alternate acceptor; AD, alternate donor; AP, alternate promoter; AT, alternate terminator; ES, exon skip; ME, mutually exclusive exons; RI, retained intron.

$\mathrm{P}<1 \times 10^{-20}$ were inputted into Cytoscape 3.5.1 (The Cytoscape Consortium, New York, NY, USA) to construct the network.

Statistical analysis. UpSet was used to visualize the intersection between genes and the 7 splicing types (29). SPSS software version 22 (IBM Corp., Armonk, NY, USA) was used for the univariate and multivariate Cox regression, Kaplan-Meier curve and Pearson correlation analyses. The tROC analysis was performed with $\mathrm{R}$ (version 3.4.3). $\mathrm{P}<0.05$ was considered to indicate a statistically significant difference. GraphPad 7.0 (GraphPad Inc., La Jolla, CA, USA) was used to plot the ROC and Kaplan-Meier curves.

\section{Results}

Comprehensive analysis of splicing events in the PRAD cohort from TCGA dataset. In the present study, a total of 44,070 splicing events were identified in the cohort of PRAD patients, including 3,524 of the AA type, 3,101 of the AD type, 9,035 of the AP type, 8,663 of the AT type, 16,772 of the ES type, 228 of the ME type and 2,747 of the RI type (Table I). UpSet, which is similar to a Venn diagram, was used to illustrate the splicing events in PRAD (Fig. 1).

Single splicing events were accurately described using the labelled name of each splicing event, which contained the gene name, splicing type and AS ID. For instance, for pleckstrin homology domain containing N1 (PLEKHN1)-ES-1, the gene name is PLEKHN1, the splicing type is ES and the AS ID is 1.

DFS-SEs in PRAD identified from TCGA. In total, 6,909 splicing events were identified as DFS-SEs by univariate Cox proportional hazards regression analysis; these included 373 of the AA type, 364 of the AD type, 1,474 of the AP type, 2,275 of the AT type, 1,956 of the ES type, 42 of the ME type and 425 of the RI type (Table I). The HRs of the top 10 splicing events for PRAD recurrence, ranked by $\mathrm{P}$-value for each splicing type, are presented in Fig. 2. Kaplan-Meier curve analyses were performed for the 10 most significant splicing events among all the DFS-SEs. Patients with PRAD were divided into 'high' and 'low' groups based on the median PSI value of a certain splicing event. The patients with PRAD who had a high PSI value for elongin B-ES-33303, FK506 binding protein 2 (FKBP2)-AP-16603, NHL repeat containing 3-ES-25701 and thioredoxin-ES-87183 also had a relatively high chance of DFS, whereas patients with high PSI values for abhydrolase domain containing 17A-AD-46558, FKBP2-AP-16602, yippee like 3-AD-36074, high mobility group AT-hook 2-AT-22879 and prostaglandin D2 synthase-AT-88235 had a reduced probability of DFS (Fig. 3).

The UpSet was also used to quantitatively display the intersection of genes and splicing types of the DFS-SEs in PRAD (Fig. 4A). Numerous splicing events of certain genes, including inositol hexakisphosphate kinase 2, mammalian target of rapamycin-associated protein, LST8 homolog and autophagy related 16 like 2, were associated with DFS. Selection of the top 500 DFS-SEs and input of their corresponding 356 genes into Cytoscape for analysis provided the gene interaction network (Fig. 4B). Of note, a total of 356 counterpart genes of the 500 most significant DFS-SEs were linked to mitochondria and associated pathways according to GO annotation, including 'mitochondrial electron transport, NADH to ubiquinone', 'mitochondrial translational elongation' and 'mitochondrial translational termination' in the category Biological Process (BP) and 'mitochondrion', 'mitochondrial inner membrane' in the category Cellular Component (CC) (Fig. 5). 


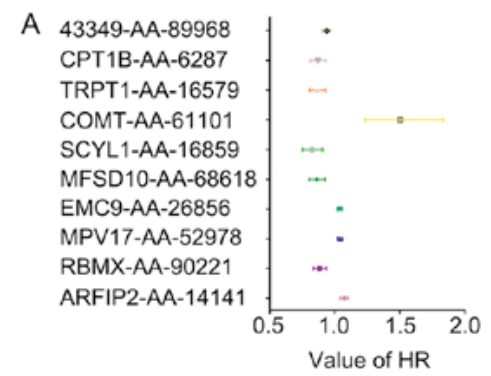

D

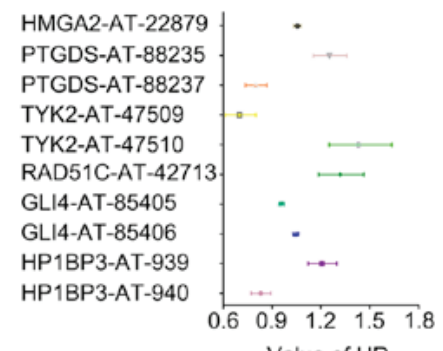

$G$

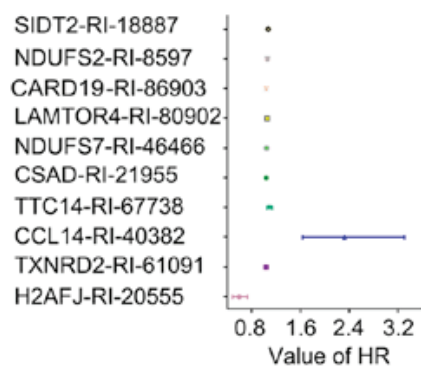

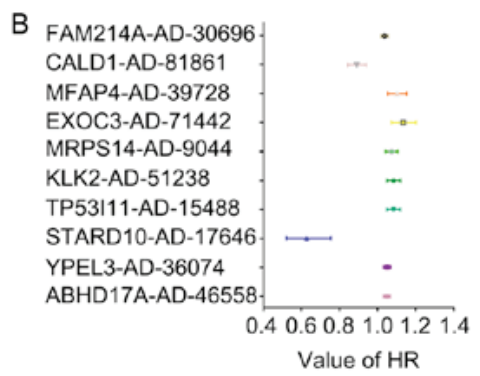

E

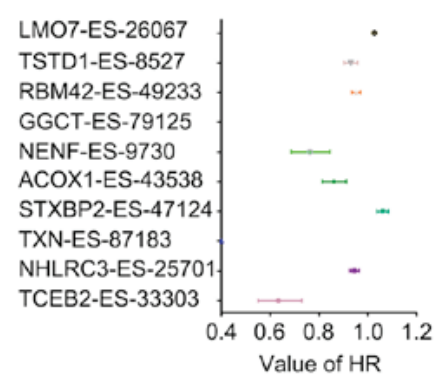

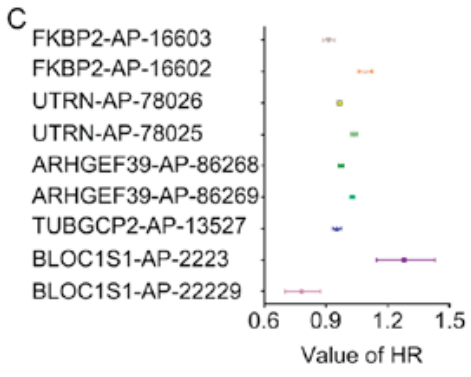

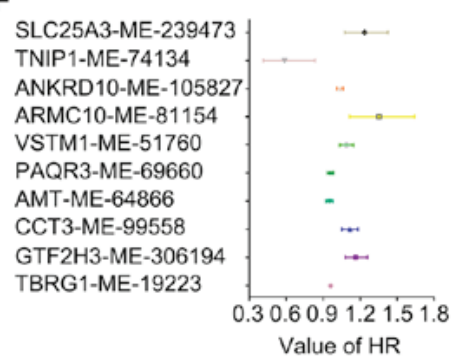

Figure 2. HRs of DFS-SEs for tumor recurrence in prostate adenocarcinoma. HRs of the top 10 DFS-SEs of (A) AA; (B) AD; (C) AP; (D) AT; (E) ES; (F) ME; and (G) RI. The horizontal lines and data-points indicate the HR with 95\% confidence interval for PRAD recurrence. HR, hazard ratio; DFS-SEs, disease-free survival-associated splicing events; AA, alternate acceptor site; AD, alternate donor site; AP, alternate promoter; AT, alternate terminator; ES, exon skip; ME, mutually exclusive exons; RI, retained intron; ARFIP2, ADP ribosylation factor interacting protein 2; RBMX, RNA binding motif protein X-linked; MPV17, mitochondrial inner membrane protein; EMC9, endoplasmic riticulum membrane protein complex subunit 9; MFSD10, major facilitator superfamily domain containing 10; SCYL1, SCY1 like pseudokinase 1; COMT, catechol-O-methyltransferase; TRPT1, tRNA phosphotransferase 1; CPT1B, carnitine palmitoyltransferase 1B; ABHD17A, abhydrolase domain containing 17A; YPEL3, yippee like 3; STARD10, StAR related lipid transfer domain containing 10; TP53I11, tumor protein p53 inducible protein 11; KLK2, kallikrein related peptidase 2; MRPS14, mitochondrial ribosomal protein S14; EXOC3, exocyst complex component 3; MFAP4, microfibril associated protein 4; CALD1, caldesmon 1; FAM214A, family with sequence similarity 214 member A; FKBP2, FK506 binding protein 2; UTRN, utrophin; ARHGEF39, Rho guanine nucleotide exchange factor 39; TMUB1, transmembrane and ubiquitin like domain containing 1; TUBGCP2, tubulin $\gamma$ complex associated protein 2; BLOC1S1, biogenesis of lysosomal organelles complex 1 subunit 1; HMGA2, high mobility group AT-hook 2; PTGDS, prostaglandin D2 synthase; TYK2, tyrosine kinase 2; RAD51C, RAD51 paralog C; GLI4, GLI family zinc finger 4; HP1BP3, heterochromatin protein 1 binding protein 3; TCEB2, elongin B; NHLRC3, NHL repeat containing 3; TXN, thioredoxin; STXBP2, syntaxin binding protein 2; ACOX1, acyl-CoA oxidase 1; NENF, neudesin neurotrophic factor; GGCT, $\gamma$-glutamylcyclotransferase; RBM42, RNA binding motif protein 42; TSTD1, thiosulfate sulfurtransferase like domain containing 1; LMO7, LIM domain 7; TBRG1, transforming growth factor $\beta$ regulator 1; GTF2H3, general transcription factor IIH subunit 3; CCT3, chaperonin containing TCP1 subunit 3; AMT, aminomethyltransferase; PAQR3, progestin and adipoQ receptor family member 3; VSTM1, V-set and transmembrane domain containing 1; ARMC10, armadillo repeat containing 10; ANKRD10, ankyrin repeat domain 10; TNIP1, tumor necrosis factor $\alpha$-induced protein 3 interacting protein 1; SLC25A3, solute carrier family 25 member 3; H2AFJ, H2A histone family member J; TXNRD2, TXN reductase 2; CCL14, C-C motif chemokine ligand 14; TTC14, tetratricopeptide repeat domain 14; CSAD, cysteine sulfinic acid decarboxylase; NDUFS7, NADH:ubiquinone oxidoreductase core subunit S7; LAMTOR4, late endosomal/lysosomal adaptor, mitogen-activated protein kinase and mammalian target of rapamycin activator 4; CARD19, caspase recruitment domain family member 19; SIDT2, SID1 transmembrane family member 2.

PI models based on the DFS-SEs in patients with PRAD. Multivariate Cox regression analyses were performed based on the top 10 significant DFS-SEs for each of the seven splicing types and for all splicing types. PIs constructed based on splicing events of the AA, AD, AP, AT, ES, ME, RI and all splicing types, and their associated data are presented in Figs. 6-13, respectively. For simplification, these eight PIs were abbreviated as PI-AA, PI-AD, PI-AP, PI-AT, PI-ES, PI-ME, PI-RI and PI-ALL, respectively. The calculation of PIs was performed according to the formula mentioned above.

Prognostic value of PIs in patients with PRAD. The tROC curve analyses indicated that PI-ME was the most effective PI at predicting the cancer status after five years, with an AUC value of 0.7606 (Fig. 13D). This was followed by PI-ALL and PI-ES, which exhibited AUC values of 0.7558 (Fig. 10E) and 0.7403 (Fig. 6E), respectively. The median values of the eight PIs were then used to classify the patients with PRAD into low- and high-level groups for the Kaplan-Meier curve analyses. The low and high groups into which the patients with PRAD were stratified based on the median value of PI-ES exhibited the most significant difference in DFS (low vs. high, $3,588.45 \pm 250.51$ vs. $1,531.08 \pm 136.50$ days; $\left.\mathrm{P}=7.43 \times 10^{-9}\right)$. After this, the most significant differences between low and high groups were those with stratification based on PI-ME and PI-AD (Table II). 

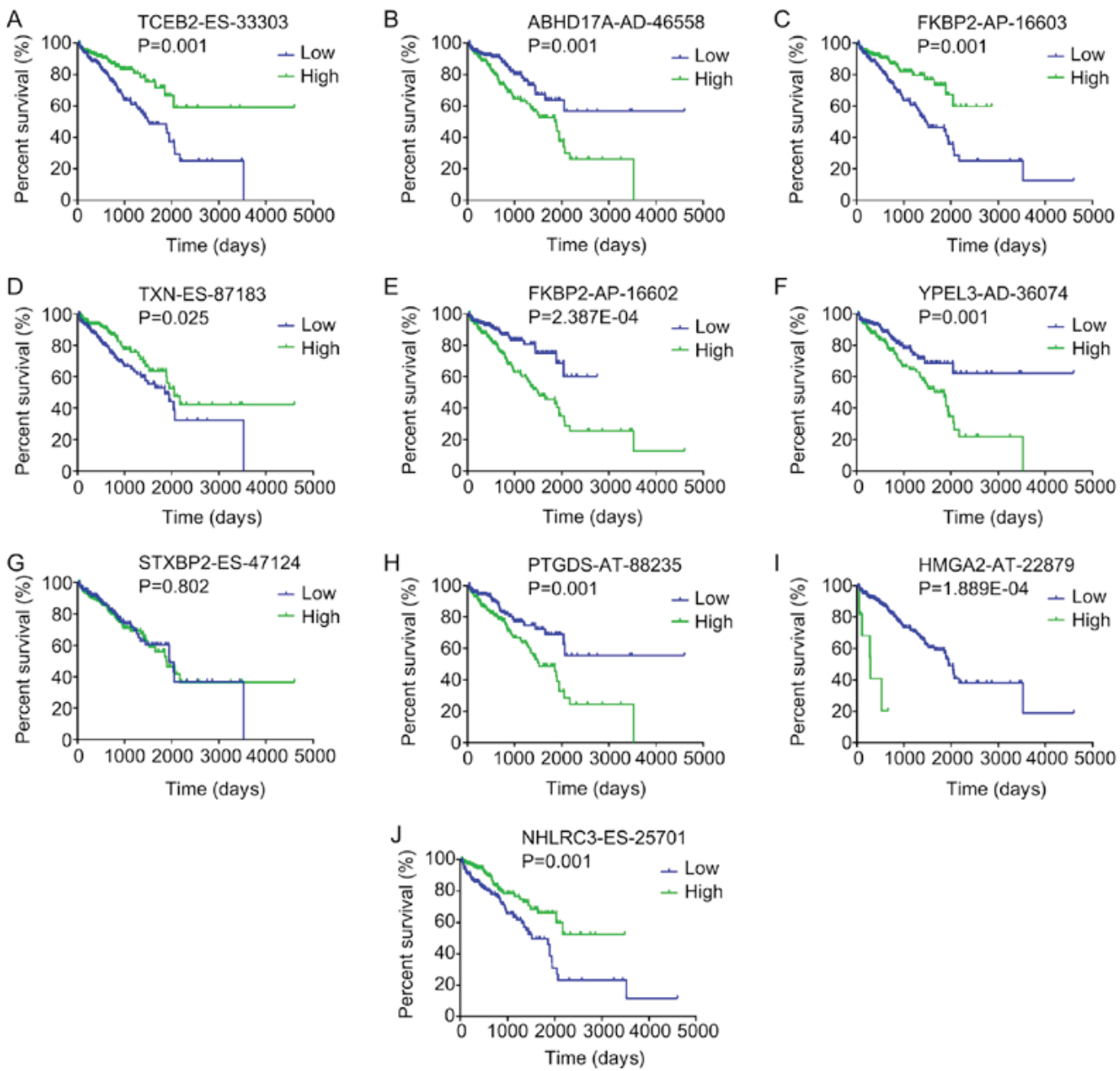

Figure 3. Kaplan-Meier curves for the top 10 most significant disease-free survival-associated splicing events in PRAD identified by univariate Cox proportional hazards regression analysis. Patients with PRAD were divided into 'high' and 'low' groups based on the median percent-spliced-in value of a certain splicing event. Kaplan-Meier curves for (A) TCEB2-ES-33303, (B) ABHD17A-AD-46558, (C) FKBP2-AP-16603, (D) TXN-ES-87183, (E) FKBP2-AP-16602, (F) YPEL3-AD-36074, (G) STXBP2-ES-47124, (H) PTGDS-AT-88235, (I) HMGA2-AT-22879 and (J) NHLRC3-ES-25701. PRAD, prostate adenocarcinoma; AA, alternate acceptor; AD, alternate donor; AP, alternate promoter; AT, alternate terminator; ES, exon skip; ME, mutually exclusive exons; RI, retained intron; TCEB2, elongin B; ABHD17A, abhydrolase domain containing 17A; FKBP2, FK506 binding protein 2; TXN, thioredoxin; YPEL3, yippee like 3; STXBP2, syntaxin binding protein 2; PTGDS, prostaglandin D2 synthase; HMGA2, high mobility group AT-hook 2; NHLRC3, NHL repeat containing 3.

The prognostic value of the PIs and other clinical parameters, including age, Gleason score, PSA value, pathologic $\mathrm{T}$ stage and pathologic $\mathrm{N}$ stage, were then analyzed using univariate Cox analysis. Except for age, the other clinical parameters and the eight PIs all had a high predictive value regarding the DFS of patients with PRAD (Table III). In addition, the multivariate analysis revealed that only the pathologic T stage, PI-AT, PI-ME and PI-ALL had an independent significant prognostic value for predicting the DFS of patients with PRAD (Table III).

Potential correlation network of DFS-SEs in PRAD. Splicing factors are key genes for the regulation of the development of splicing events (30). Splicing factors also have their own corresponding splicing events. A correlation analysis was used to examine the potential regulatory association between the splicing events of splicing factors and non-splicing factors and to construct a regulatory network.

A total of 66 splicing factors were obtained from the SpliceAid2 database. Based on the univariate Cox analysis,
22 splicing events of the 66 splicing factors with $\mathrm{P}<0.01$ were selected, as well as the most significant top 50 splicing events of non-splicing factors with $\mathrm{P}<2.5 \times 10^{-06}$. The correlations between the PSI values of the 22 splicing events of the splicing factors and the PSI values of the top 50 splicing events of nonsplicing factors were calculated. Splicing events with $\mathrm{P}<1 \times 10^{-20}$ according to the correlation analysis were then introduced into Cytoscape to generate the network (Fig. 14A). The red nodes in the graph indicate the splicing events of the splicing factors $(n=17)$ and the light blue nodes indicate the splicing events of non-splicing factors $(n=25)$. The triangular nodes indicate the splicing events associated with good prognosis and the oval nodes indicate the splicing events associated with poor prognosis. The blue lines indicate negative correlations between two splicing events and the green lines indicate positive correlations between two splicing events.

The correlation network revealed a complex association between the splicing events of splicing and non-splicing factors. For instance, the splicing event of splicing factor serine and arginine-rich splicing factor 2-RI-43660 was positively 


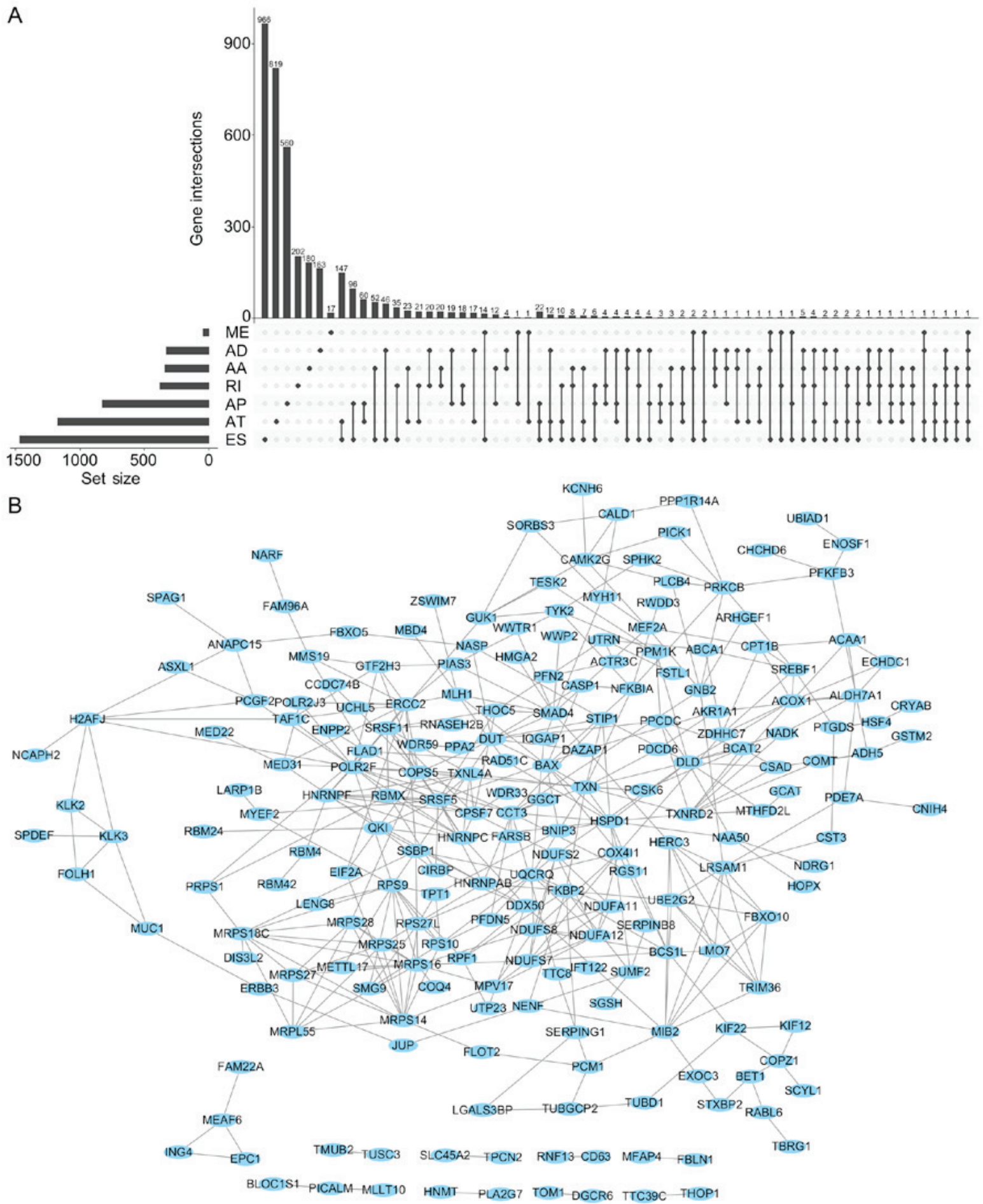

Figure 4. UpSet plots of DSEs and gene interaction network in PRAD. (A) UpSet plots of DSEs in PRAD. (B) Gene interaction network based on counterpart genes of the top 500 DSEs in PRAD. DSEs, disease-free survival-associated splicing events; PRAD, prostate adenocarcinoma; AA, alternate acceptor; $\mathrm{AD}$, alternate donor; AP, alternate promoter; $\mathrm{AT}$, alternate terminator; ES, exon skip; ME, mutually exclusive exons; RI, retained intron.

correlated with ectonucleoside triphosphate diphosphohydrolase 5 (ENTPD5)-AT-28353 and was negatively correlated with ENTPD5-AT-28354. Of note, the splicing events with good DFS were predominantly negatively correlated with the splicing events of the splicing factors, whereas the splicing events with poor DFS were predominantly positively correlated with the splicing events of the splicing factors (Fig. 14A). Fig. 14B and C also present two pairs of splicing events of splicing and non-splicing factors with the most significant positive and negative correlations among all combinations and their Kaplan-Meier curve analyses. The PSI values of RNA binding motif protein 5 (RBM5)-AT-64957 and heterochromatin protein 1 binding protein 3 (HP1BP3)-AT-939 were negatively correlated (Fig. 14B), while the PSI values of RBM5-AT-64955 and HP1BP3-AT-939 were positively correlated (Fig. 14C). Patients with PRAD were then divided into low and high groups based on the median PSI value of certain splicing events. PRAD patients in the low group 


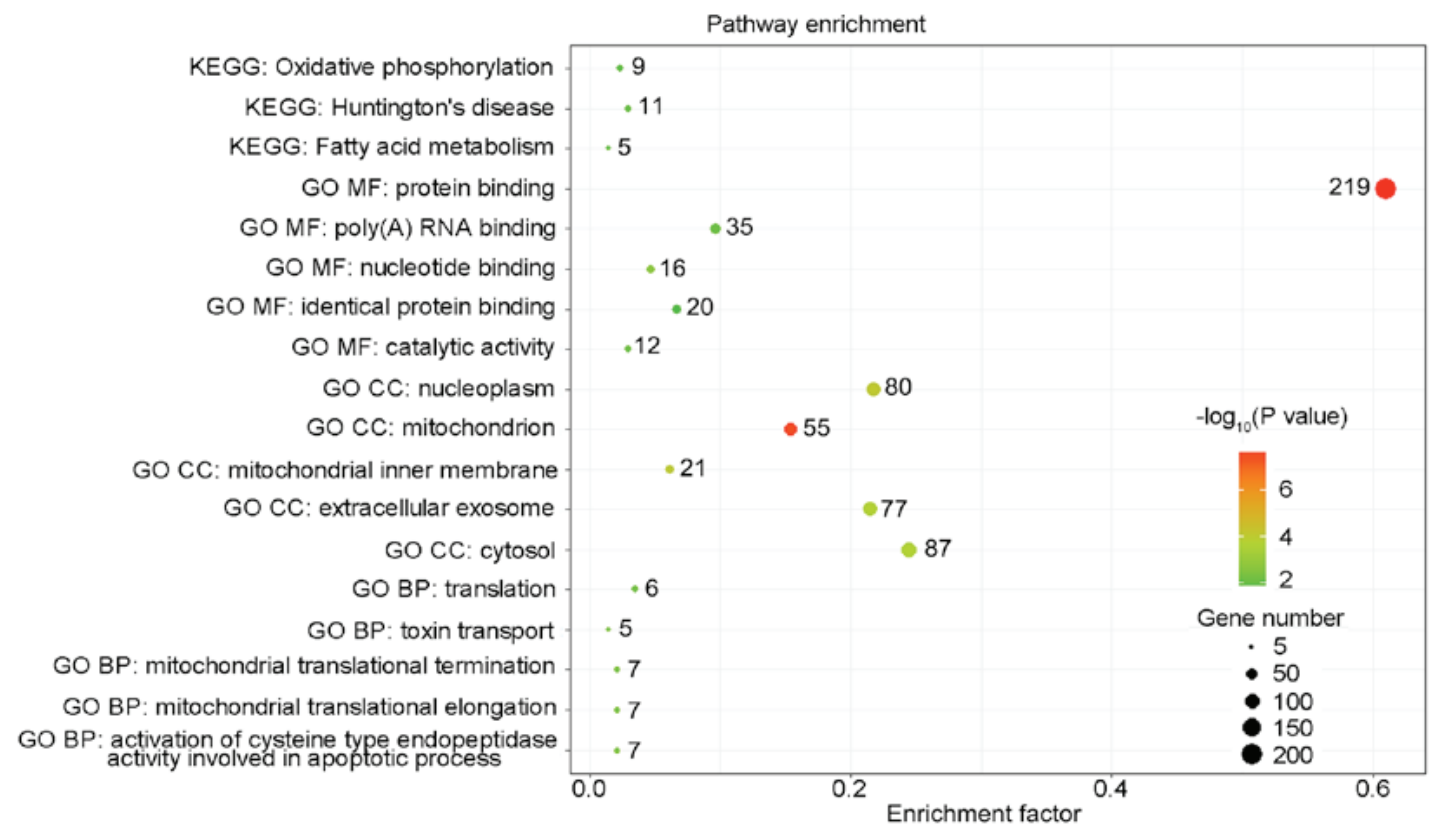

Figure 5. Pathways identified by GO and KEGG analyses. The counterpart genes of the top 500 disease-free survival-associated splicing events were subjected to GO and KEGG pathway analyses. The number of enriched genes for each term/pathway is indicated by the respective data-points. MF, molecular function; CC, cellular component; BP, biological process; KEGG, Kyoto Encyclopedia of Genes and Genomes; GO, Gene Ontology.

A

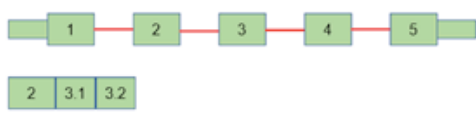

\begin{tabular}{|l|l|l|l}
\hline 2 & 32 & Alternate acceptor (AA)
\end{tabular}
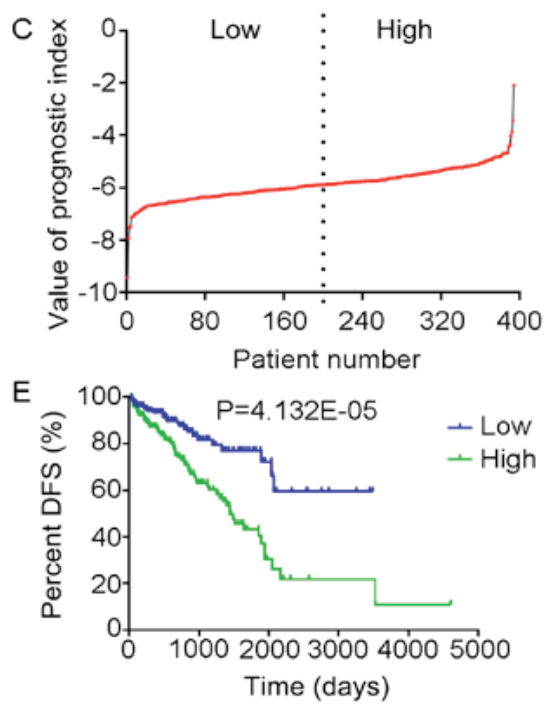
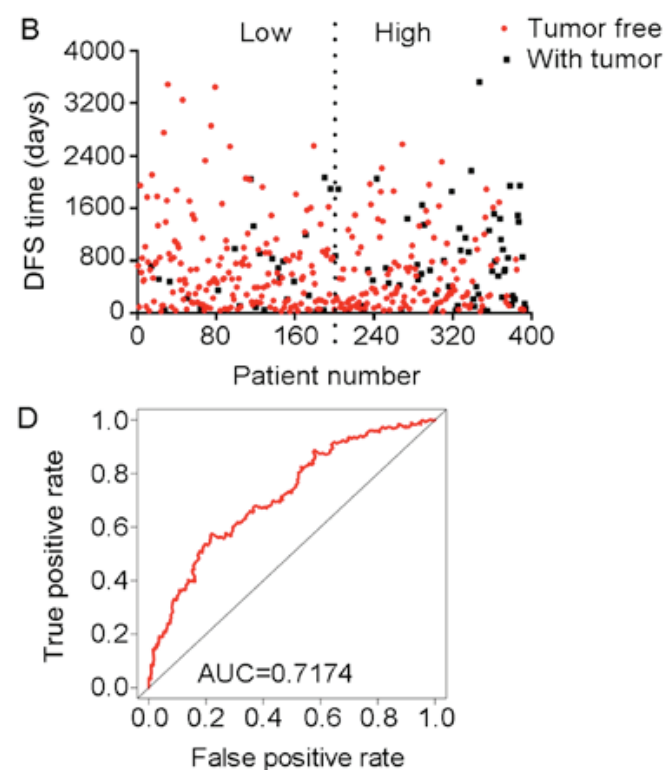

Figure 6. Analysis of the PI-AA. The three splicing events of AA, from which the PI-AA was generated, were identified from DFS-associated splicing events by multivariate Cox regression analysis. (A) Illustration of AA site. (B) Scatter plot of time of DFS vs. cancer status of PRAD patients. (C) PI value curve for patients with PRAD. (D) Time-dependent receiver-operator characteristic curve of PI for predicting the tumor status after five years. (E) Kaplan-Meier curve of PI for evaluating the DFS time of PRAD patients stratified by median PI value into low and high group. PI-AA, prognostic index based on Alternate Acceptor site type of splicing events; PRAD, prostate adenocarcinoma; AUC, area under curve; DFS, disease-free survival; ARFIP2, ADP ribosylation factor interacting protein 2; RBMX, RNA binding motif protein X-linked; COMT, catechol-O-methyltransferase.

of RBM5-AT-64957 had a significantly higher DFS than those in the high group (Fig. 14D), while PRAD patients in the high RBM5-AT-64955 or HP1BP3-AT-939 group had a markedly higher DFS than those in the respective low group (Fig. 14E and F).

\section{Discussion}

AS is an important regulatory mechanism of gene expression, and abnormalities of this mechanism may cause the development of various diseases (14), including the entirety of the 
A

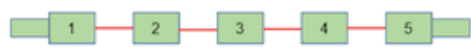

$21|22| 3$

\begin{tabular}{l|l|l|l}
21 & 3 & Alternate donor site $(A D)$
\end{tabular}

C
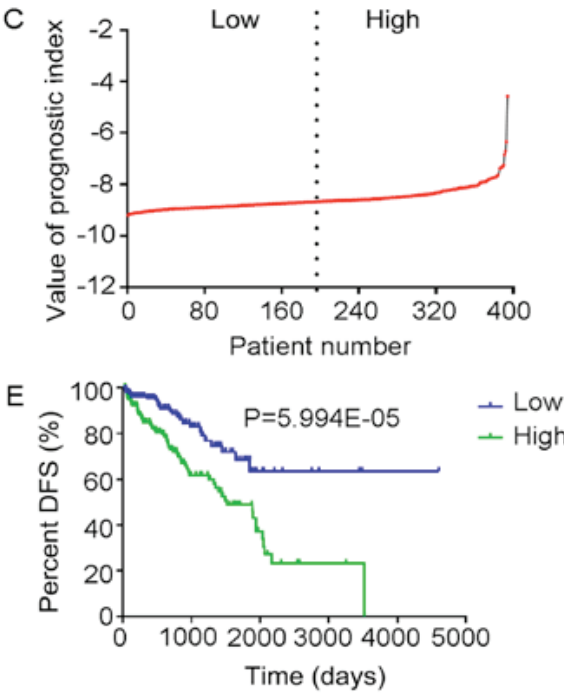

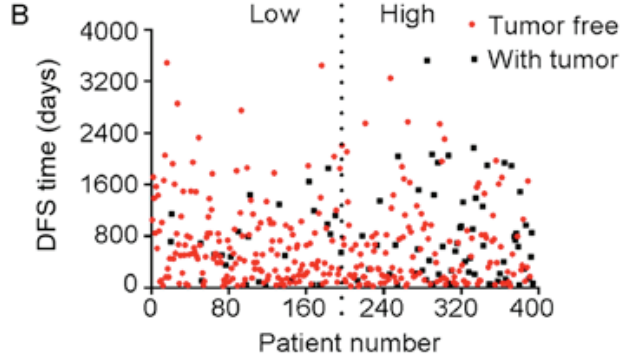

$\mathrm{D}$

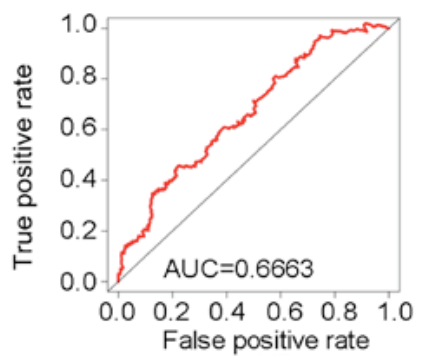

Figure 7. Analysis of the PI-AD. The three splicing events of AD, from which the PI-AD was generated, were identified from DFS-associated splicing events by multivariate Cox regression analysis. (A) Illustration of AD site. (B) Scatter plot of time of DFS vs. cancer status of PRAD patients. (C) PI value curve for patients with PRAD. (D) Time-dependent receiver-operator characteristic curve of PI for predicting the tumor status after five years. (E) Kaplan-Meier curve of PI for evaluating the DFS time of PRAD patients stratified by median PI value into low and high group. PI-AD, prognostic index based on the Alternate Donor site type of splicing events; PRAD, prostate adenocarcinoma; AUC, area under curve; DFS, disease-free survival; YPEL3, yippee like 3; KLK2, kallikrein related peptidase 2; CALD1, caldesmon 1 .

A
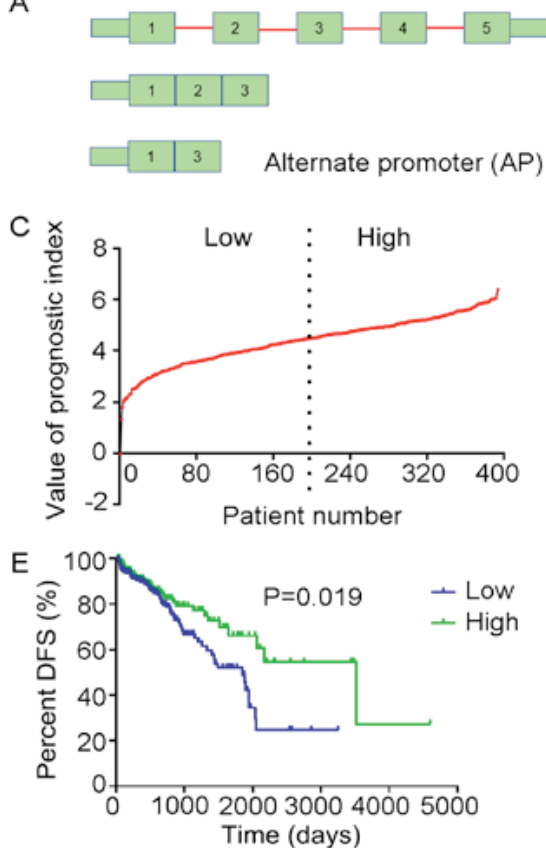

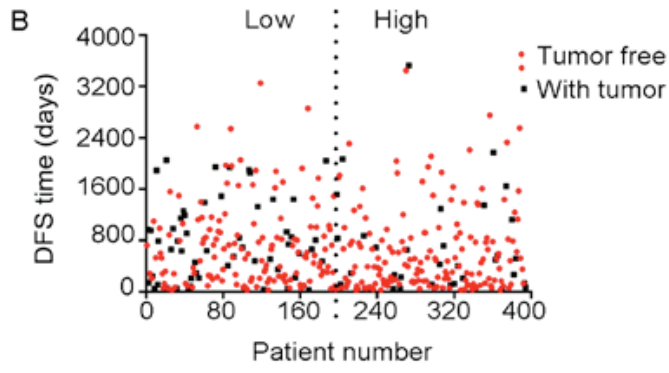

D

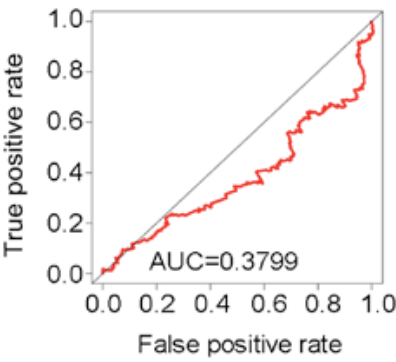

Figure 8. Analysis of PI-AP. The three splicing events of the AP type from which the PI-AP was generated were identified from DFS-associated splicing events by multivariate Cox regression analysis. (A) Illustration of AP site. (B) Scatter plot of time of DFS vs. cancer status of PRAD patients. (C) PI value curve for patients with PRAD. (D) Time-dependent receiver-operator characteristic curve of PI for predicting the tumor status after five years. (E) Kaplan-Meier curve of PI for evaluating the DFS time of PRAD patients stratified by median PI value into low and high group. PI-AP, prognostic index based on the Alternate Promoter type of splicing events; PRAD, prostate adenocarcinoma; AUC, area under curve; DFS, disease-free survival; FKBP2, FK506 binding protein 2; ARHGEF39, Rho guanine nucleotide exchange factor 39; BLOC1S1, biogenesis of lysosomal organelles complex 1 subunit 1.

processes of cancer development and progression. Certain products of AS may therefore be used as diagnostic and prognostic indicators, as well as therapeutic targets for cancer $(14,20)$. A small number of studies have been published on the use AS in 
A

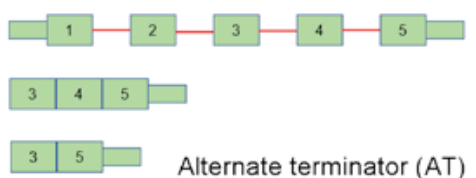

C

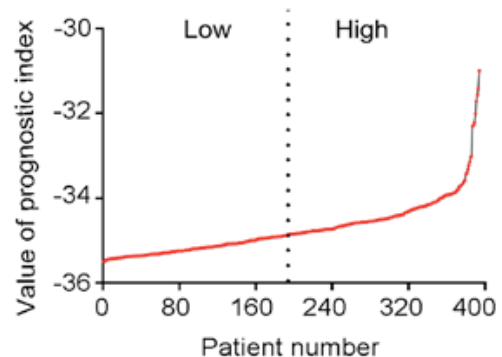

E

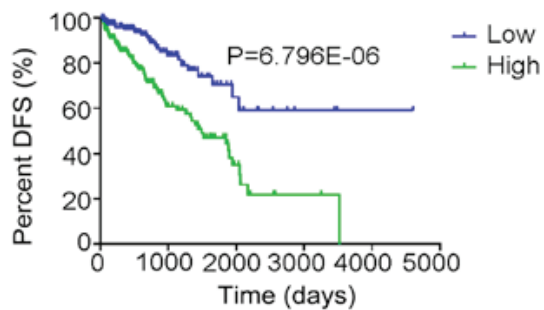

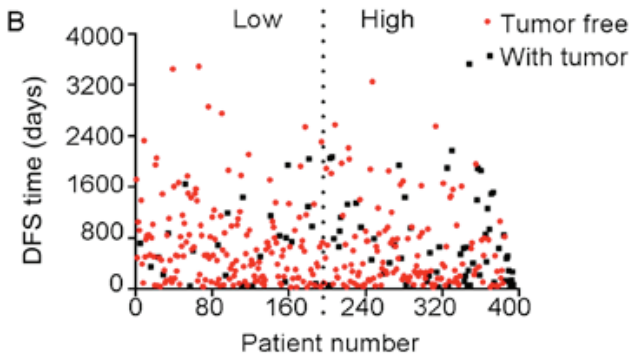

D

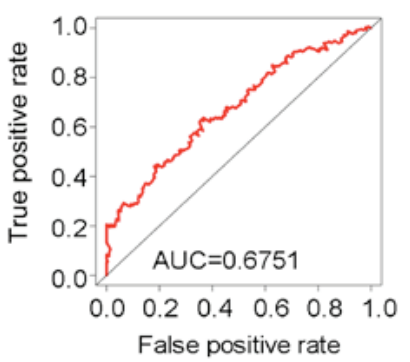

Figure 9. Analysis of PI-AT. The four splicing events of the AT type from which the PI-AT was generated, were identified from DFS-associated splicing events by multivariate Cox regression analysis. (A) Illustration of AT site. (B) Scatter plot of time of DFS vs. cancer status of PRAD patients. (C) PI value curve for patients with PRAD. (D) Time-dependent receiver-operator characteristic curve of PI for predicting the tumor status after five years. (E) Kaplan-Meier curve of PI for evaluating the DFS time of PRAD patients stratified by median PI value into low and high group. PI-AT, prognostic index based on the Alternate Terminator type of splicing events; PRAD, prostate adenocarcinoma; AUC, area under curve; DFS, disease-free survival; HMGA2, high mobility group AT-hook 2; PTGDS, prostaglandin D2 synthase; TYK2, tyrosine kinase 2; RAD51C, RAD51 paralog C.

A

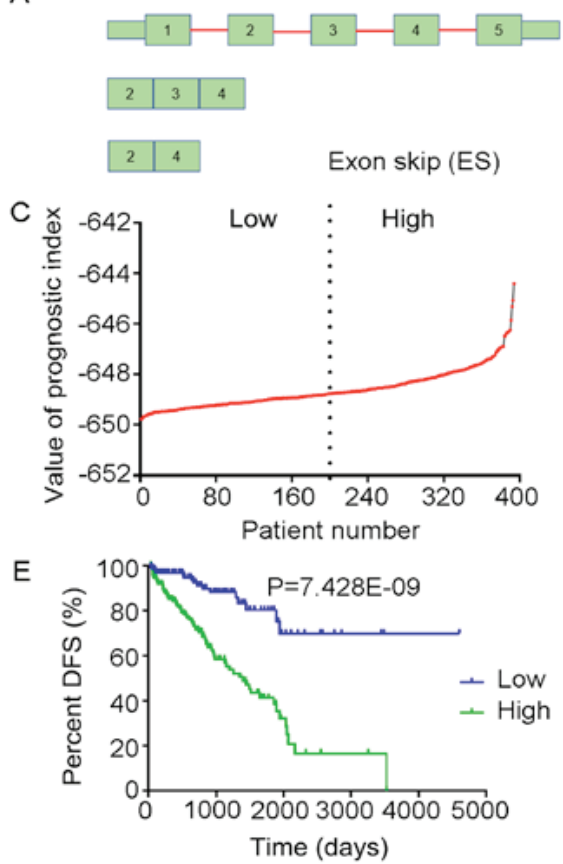

B 4000 Low : High. Tumor free

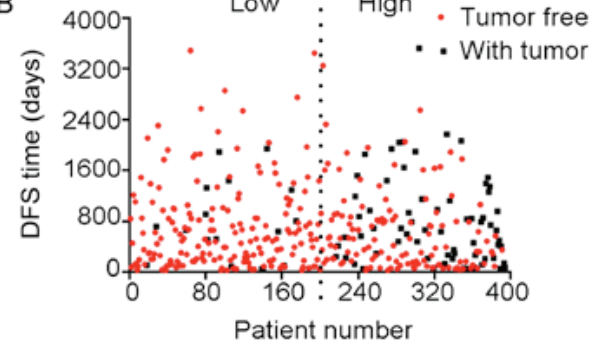

D

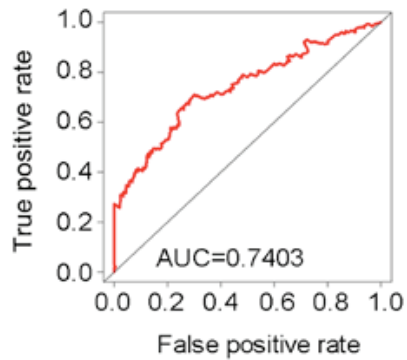

Figure 10. Analysis of PI-ES. The four splicing events of the ES type from which the PI-ES was generated, were identified from DFS -associated splicing events by multivariate Cox regression analysis. (A) Illustration of ES site. (B) Scatter plot of time of DFS vs. cancer status of PRAD patients. (C) PI value curve for patients with PRAD. (D) Time-dependent receiver-operator characteristic curve of PI for predicting the tumor status after five years. (E) Kaplan-Meier curve of PI for evaluating the DFS time of PRAD patients stratified by median PI value into low and high group. PI-ES, prognostic index based on the Exon Splice type of splicing events; PRAD, prostate adenocarcinoma; AUC, area under curve; DFS, disease-free survival; TCEB2, elongin B; TXN, thioredoxin; GGCT, $\gamma$-glutamylcyclotransferase; LMO7, LIM domain 7.

PRAD, but these have primarily focused on splicing variants of the androgen receptor (14,15,31-33), proprotein convertase subtilisin/kexin type 6 (34), CD44 (35) and staphylococcal nuclease and tudor domain containing 1 (36). However, studies 
A

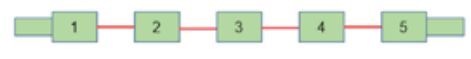

\begin{tabular}{l|l|l|l|l|l}
\hline 2 & 3 & 4 \\
\hline
\end{tabular}

\begin{tabular}{|l|l|l|l|l}
2 & 4 & 5 & Mutually exclusive exons (ME) \\
\hline
\end{tabular}

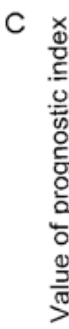

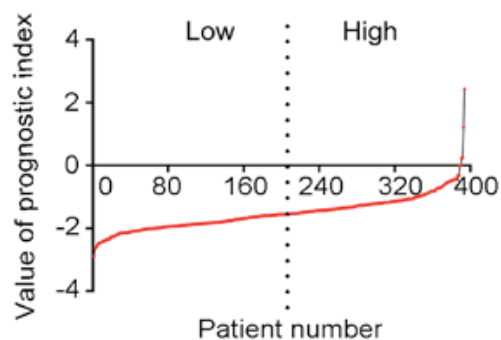

E

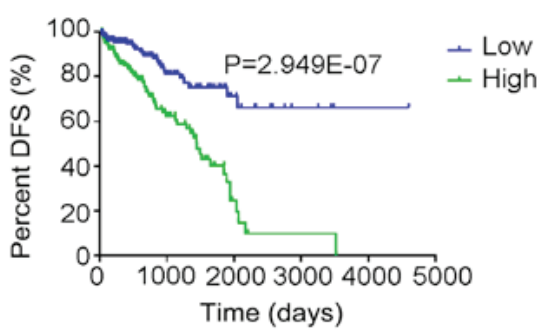

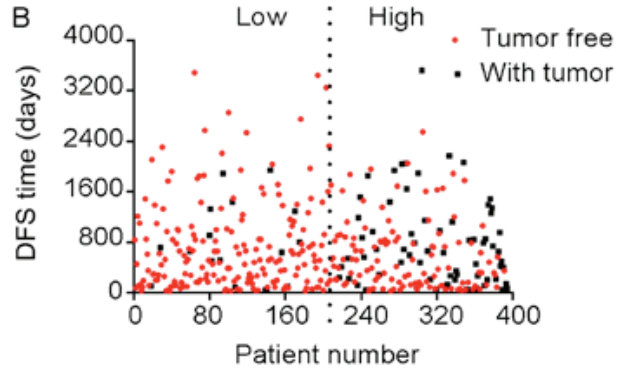

D

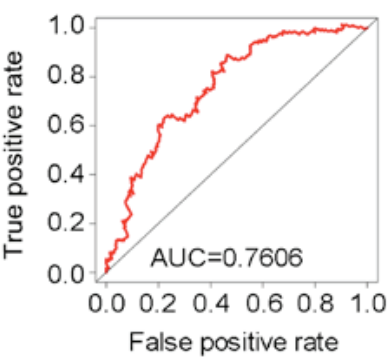

Figure 11. Analysis of PI-ME. The five splicing events of the ME type from which the PI-ME was generated, were identified from DFS-associated splicing events by multivariate Cox regression analysis. (A) Illustration of ME site. (B) Scatter plot of time of DFS vs. cancer status of PRAD patients. (C) PI value curve for patients with PRAD. (D) Time-dependent receiver-operator characteristic curve of PI for predicting the tumor status after five years. (E) KaplanMeier curve of PI for evaluating the DFS time of PRAD patients stratified by median PI value into low and high group. PI-ME, prognostic index based on the Mutually Exclusive Exons type of splicing events; PRAD, prostate adenocarcinoma; AUC, area under curve; DFS, disease-free survival; TBRG1, transforming growth factor $\beta$ regulator 1; GTF2H3, general transcription factor IIH subunit 3; CCT3, chaperonin containing TCP1 subunit 3; VSTM1, V-set and transmembrane domain containing 1; ANKRD10, ankyrin repeat domain 10.

A

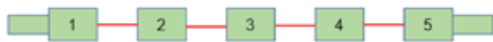
$2 \mid$\begin{tabular}{l|l|l}
2 & 3 & 4 \\
\hline
\end{tabular}
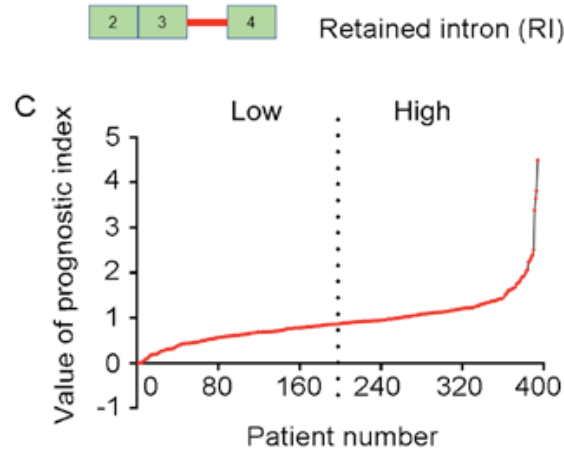

$\mathrm{E}$

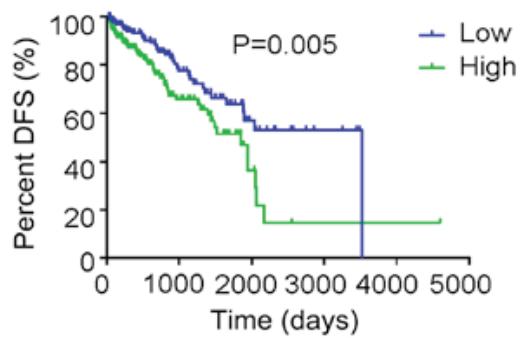

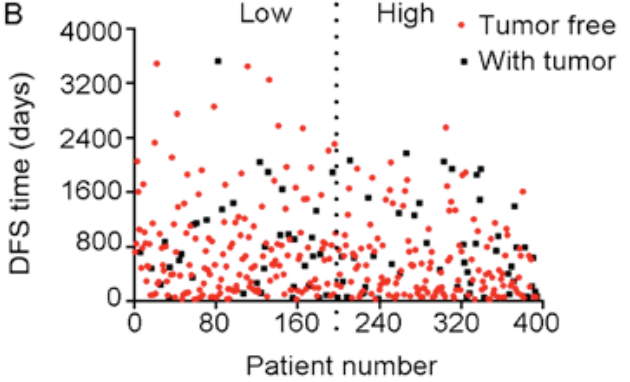

D

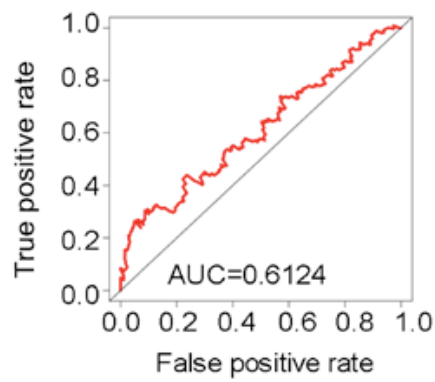

Figure 12. Analysis of PI-RI. The two splicing events of the RI type from which the PI-RI was generated, were identified from DFS-associated splicing events by multivariate Cox regression analysis. (A) Illustration of RI site. (B) Scatter plot of time of DFS vs. cancer status of PRAD patients. (C) PI value curve for patients with PRAD. (D) Time-dependent receiver operating characteristic curve of PI for predicting the tumor status after five years. (E) Kaplan-Meier curve of PI for evaluating the DFS time of PRAD patients stratified by median PI value into low and high group. PI-RI, prognostic index based on the Retained Intron type of splicing events; PRAD, prostate adenocarcinoma; AUC, area under curve; DFS, disease-free survival; CCL14, C-C motif chemokine ligand 14; CSAD, cysteine sulfinic acid decarboxylase. 
Table I. Overview of the splicing events in prostate adenocarcinoma (n).

\begin{tabular}{lccccr}
\hline & \multicolumn{2}{c}{ Total splicing events } & & \multicolumn{2}{c}{ DFS-SEs } \\
\cline { 2 - 3 } \cline { 5 - 6 } Type & Splicing events & Genes & & Splicing events & Genes \\
& & & & & \\
AA & 3524 & 2488 & & 373 & 341 \\
AD & 3101 & 2185 & & 364 & 325 \\
AP & 9035 & 3621 & & 1474 & 826 \\
AT & 8663 & 3781 & & 2275 & 1171 \\
ES & 16772 & 6577 & & 1956 & 1466 \\
ME & 228 & 221 & & 42 & 42 \\
RI & 2747 & 1849 & & 425 & 375 \\
Total & 44070 & 10380 & 6909 & 3645 \\
\hline
\end{tabular}

DFS-SEs, disease-free survival-associated splicing events; AA, alternate acceptor; $\mathrm{AD}$, alternate donor; $\mathrm{AP}$, alternate promoter; $\mathrm{AT}$, alternate terminator; ES, exon skip; ME, mutually exclusive exons; $\mathrm{RI}$, retained intron.

providing a comprehensive evaluation of splicing events in PRAD are scarce.

In the present study, splicing events in PRAD were comprehensively evaluated based on data from the TCGASpliceSeq database. In those analyses, the total number of splicing events greatly exceeded the number of genes, indicating that AS markedly increases the diversity of gene expression products in PRAD, as has been observed in other diseases $(14,20,37)$. One of the highlights of the present study was the comprehensive and systematic presentation of all splicing events and DFS-SEs in PRAD, which may provide clues for studying the functions of AS and its products in PRAD.

The present study also analyzed the functions of genes corresponding to DFS-SEs in PRAD, indicating that these genes were mainly enriched in the fatty acid (FA) metabolism and oxidative phosphorylation pathways. An increasing number of cohort studies and experiments have confirmed a close association between FA metabolism and PRAD. For instance, a cohort study by Brasky et al (38) indicated an increased risk for the development of PRAD in males who had high blood concentrations of long-chain $\omega-3$ polyunsaturated (PU)FAs $(20: 5 \omega 3 ; 22: 5 \omega 3 ; 22: 6 \omega 3)$. The risk of PRAD was also positively correlated with the percentage of plasma phospholipids and saturated FAs. In addition, dietary n-6 PU fats, primarily linoleic acids, were significantly associated with an increased risk of PRAD (39). Among low-risk PRAD patients, the $\omega-3$ PUFAs, particularly eicosapentaenoic acid, in prostate tissues may be a protective factor against PRAD (40).

In addition to risk prediction, FA metabolism also appears to have an important role in the survival and prognosis of patients with PRAD. De novo FA synthesis is important for the survival and progression of PRAD cells. FA synthase, a key enzyme in FA synthesis, is frequently highly expressed in human PRAD, and its expression is closely associated with poor prognosis and low survival rates (41). In vitro, treatment with phenethyl isothiocyanate inhibited the oxidative phosphorylation activity in LNCaP and PC-3 human prostate
Table II. Analysis of Kaplan-Meier curves of PI for evaluating the DFS time of patients with prostate adenocarcinoma stratified by the PI value (cutoff at median).

\begin{tabular}{|c|c|c|}
\hline Type/group & DFS time (days) & P-value \\
\hline \multicolumn{3}{|l|}{ PI-AA } \\
\hline Low $(<-5.88)$ & $2583.742 \pm 174.316$ & $4.13 \times 10^{-5}$ \\
\hline $\operatorname{High}(\geq-5.88)$ & $1778.112 \pm 200.024$ & \\
\hline \multicolumn{3}{|l|}{ PI-AD } \\
\hline Low $(<-8.67)$ & $3306.887 \pm 246.458$ & $5.99 \times 10^{-5}$ \\
\hline $\operatorname{High}(\geq-8.67)$ & $1704.737 \pm 145.898$ & \\
\hline \multicolumn{3}{|l|}{ PI-AP } \\
\hline Low $(<4.89)$ & $1721.248 \pm 135.488$ & $1.95 \times 10^{-2}$ \\
\hline High ( $\geq 4.89)$ & $2741.437 \pm 305.572$ & \\
\hline \multicolumn{3}{|l|}{ PI-AT } \\
\hline Low $(<-34.84)$ & $3223.958 \pm 272.204$ & $6.80 \times 10^{-6}$ \\
\hline High $(\geq-34.84)$ & $1659.270 \pm 149.443$ & \\
\hline \multicolumn{3}{|l|}{ PI-ES } \\
\hline Low $(<-648.78)$ & $3588.446 \pm 250.513$ & $7.43 \times 10^{-9}$ \\
\hline High $(\geq-648.78)$ & $1531.083 \pm 136.504$ & \\
\hline \multicolumn{3}{|l|}{ PI-ME } \\
\hline Low $(<-1.56)$ & $3412.259 \pm 231.568$ & $2.95 \times 10^{-7}$ \\
\hline $\operatorname{High}(\geq-1.56)$ & $1450.951 \pm 129.526$ & \\
\hline \multicolumn{3}{|l|}{ PI-RI } \\
\hline Low $(<0.87)$ & $2395.445 \pm 160.811$ & $4.59 \times 10^{-3}$ \\
\hline High $(\geq 0.87)$ & $1784.987 \pm 247.797$ & \\
\hline \multicolumn{3}{|l|}{ PI-ALL } \\
\hline Low $(<-491.40)$ & $2751.999 \pm 171.559$ & $1.45 \times 10^{-7}$ \\
\hline High $(\geq-491.40)$ & $1700.247 \pm 184.410$ & \\
\hline
\end{tabular}

PI, prognostic index; DFS, disease-free survival; PI-ALL, PI based on all seven types of splicing events; PI-AA, PI based on AA type of splicing events; AA, alternate acceptor; AD, alternate donor; AP, alternate promoter; AT, alternate terminator; ES, exon skip; ME, mutually exclusive exons; RI, retained intron.

cancer cells and promoted the production of reactive oxygen species to induce cancer cell death (42).

Of note, the GO analysis of the present study indicated that the genes involved in CC and BP pathways were mainly enriched in the mitochondria and their associated pathways. The mitochondrion is the major location of cellular FA metabolism and oxidative phosphorylation (43). These results were consistent with those of the KEGG analysis, which indicated enrichment of the DFS-SEs in these two pathways and further supported the accuracy and reliability of the present bioinformatics analyses.

In the present study, splicing variants were indicated to have prognostic value in patients with PRAD. The eight PIs were constructed based on DFS-SEs without taking the therapeutic strategies, including targeted molecular therapy and radiation therapy, into account, which were excluded by a multivariate Cox analysis (data not shown). tROC analysis indicated that in patients with PRAD, the AUC of PI-ES 
Table III. Logistic regression analysis of the association of clinical parameters and the PIs with the risk of prostate adenocarcinoma recurrence.

\begin{tabular}{|c|c|c|c|c|}
\hline \multirow[b]{2}{*}{ Clinical feature } & \multicolumn{2}{|c|}{ Univariate Cox analysis } & \multicolumn{2}{|c|}{ Multivariate Cox analysis } \\
\hline & $\mathrm{HR}(95 \% \mathrm{CI})$ & P-value & $\mathrm{HR}(95 \% \mathrm{CI})$ & P-value \\
\hline Age ( $\geq 61$ vs. $<61$ years $)$ & $1.463(0.952-2.246)$ & $8.20 \times 10^{-2}$ & $1.103(0.663-1.834)$ & $7.06 \times 10^{-1}$ \\
\hline Gleason score ( $\geq 8$ vs. $<8$ ) & $4.515(2.738-7.444)$ & $3.47 \times 10^{-9}$ & $1.429(0.689-2.961)$ & $3.38 \times 10^{-1}$ \\
\hline PSA value ( $\geq 0.1$ vs. $<0.1)$ & $5.587(3.451-9.043)$ & $2.54 \times 10^{-12}$ & $2.946(1.702-5.099)$ & $1.14 \times 10^{-4}$ \\
\hline Pathologic T stage (T3/T4 vs. T1/T2) & $7.010(3.233-15.199)$ & $8.17 \times 10^{-7}$ & $4.180(1.403-12.450)$ & $1.00 \times 10^{-1}$ \\
\hline Pathologic N stage (N1 vs. N0) & $2.635(1.666-4.166)$ & $3.42 \times 10^{-5}$ & $1.024(0.589-1.780)$ & $9.34 \times 10^{-1}$ \\
\hline PI-AA ( $\geq-5.88$ vs. $<-5.88)$ & $2.531(1.598-4.008)$ & $7.57 \times 10^{-8}$ & $1.588(0.847-2.980)$ & $1.50 \times 10^{-1}$ \\
\hline PI-AD ( $\geq-8.67$ vs. $<-8.67)$ & $2.479(1.567-3.922)$ & $1.05 \times 10^{-4}$ & $0.942(0.502-1.769)$ & $8.53 \times 10^{-1}$ \\
\hline PI-AP ( $\geq 4.89$ vs. $<4.89)$ & $0.578(0.370-0.902)$ & $1.60 \times 10^{-2}$ & $1.080(0.600-1.946)$ & $7.97 \times 10^{-1}$ \\
\hline PI-AT ( $\geq-34.84$ vs. $<-34.84)$ & $2.604(1.668-4.065)$ & $2.54 \times 10^{-5}$ & $1.173(0.635-2.167)$ & $6.11 \times 10^{-1}$ \\
\hline PI-ES ( $\geq-648.78$ vs. $<-648.78$ ) & $4.097(2.439-6.881)$ & $9.77 \times 1^{0-8}$ & $2.599(1.329-5.084)$ & $5.00 \times 10^{-3}$ \\
\hline PI-ME ( $\geq-1.56$ vs. $<-1.56)$ & $3.152(1.985-5.004)$ & $1.12 \times 10^{-6}$ & $1.775(0.999-3.155)$ & $5.00 \times 10^{-2}$ \\
\hline PI-RI ( $\geq 0.87$ vs. $<0.87$ ) & $1.836(1.199-2.811)$ & $5.00 \times 10^{-3}$ & $0.624(0.337-1.157)$ & $1.34 \times 10^{-1}$ \\
\hline PI-ALL ( $\geq-491.40$ vs. $<-491.40)$ & $3.512(2.131-5.789)$ & $8.28 \times 10^{-7}$ & $1.273(0.641-2.528)$ & $4.91 \times 10^{-1}$ \\
\hline
\end{tabular}

HR, hazard ratio; CI, confidence interval; PSA, prostate-specific antigen; PI, prognostic index; PI-ALL, PI based on all seven types of splicing events; PI-AA, PI based on AA type of splicing events; AA, alternate acceptor; AD, alternate donor; AP, alternate promoter; AT, alternate terminator; ES, exon skip; ME, mutually exclusive exons; RI, retained intron.
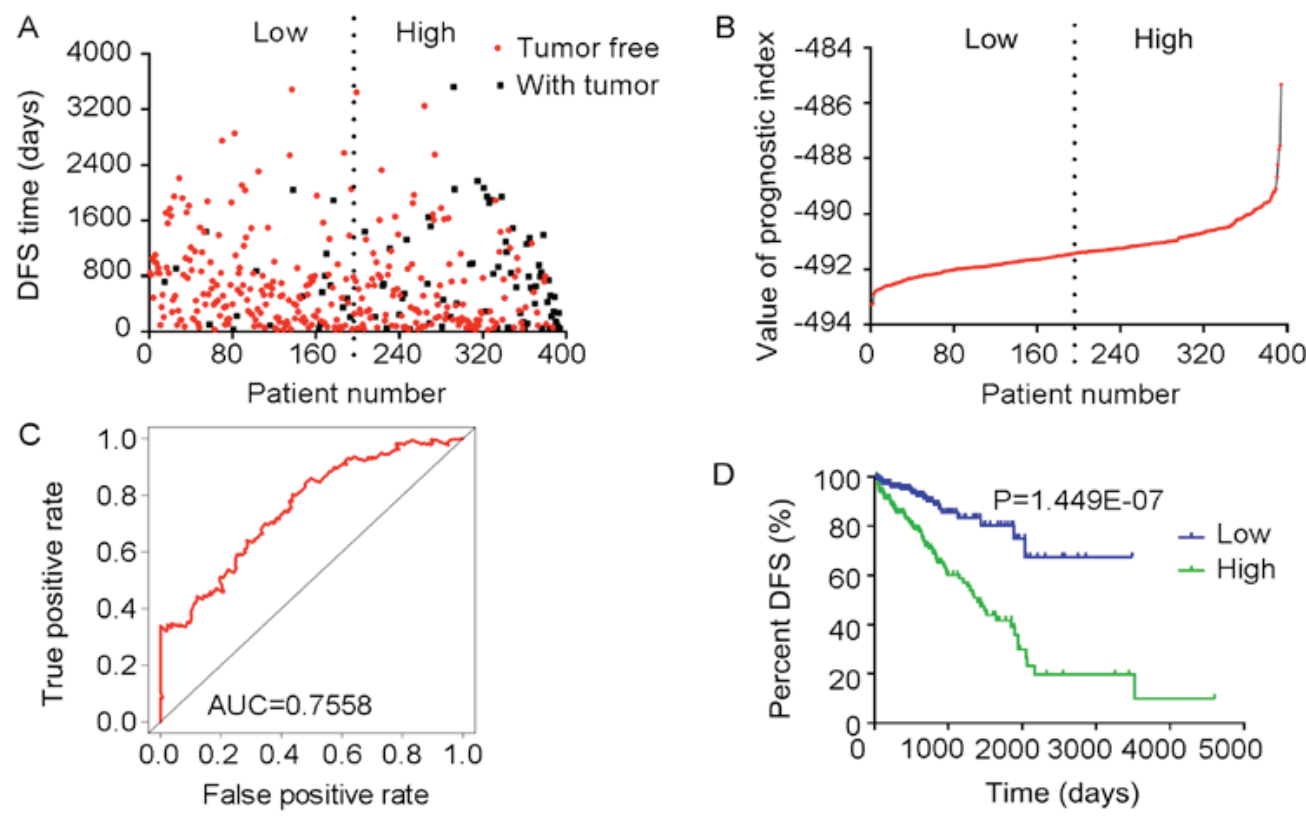

Figure 13. Analysis of PI-ALL. The five splicing events from which the PI-ALL was generated were identified from DFS-associated splicing events by multivariate Cox regression analysis. (A) Scatter plot of time of DFS vs. cancer status of PRAD patients. (B) PI value curve for patients with PRAD. (C) Time-dependent receiver-operator characteristic curve of PI for predicting the tumor status after five years; (D) Kaplan-Meier curve of PI for evaluating the DFS time of PRAD patients stratified by median PI value into low and high group. PI-ALL, prognostic index based on all seven types of splicing events; PRAD, prostate adenocarcinoma; AUC, area under curve; DFS, disease-free survival; NHLRC3, NHL repeat containing 3; TXN, thioredoxin; FKBP2, FK506 binding protein 2; HMGA2, high-mobility group AT-hook 2; STXBP2, syntaxin binding protein 2.

was 0.7403 for predicting the cancer status after five years. In addition, the HR of PI-ES for tumor recurrence was 4.097 (95\% CI: $2.439-6.881, \mathrm{P}=9.77 \times 10^{-8}$ ) according to univariate Cox analysis and 2.599 (95\% CI: 1.329-5.084, $\mathrm{P}=0.005$ ) according to multivariate Cox analysis, which were better than previously reported values focusing on only a single indicator, including programmed death-1 receptor methylation (44), pituitary homeobox 3 methylation (45) and cysteine dioxygenase 1 promoter methylation (46).

The functions of different transcripts of the same gene may differ. Therefore, an increasing number of studies have been assessing the functions of specific mature mRNAs or 

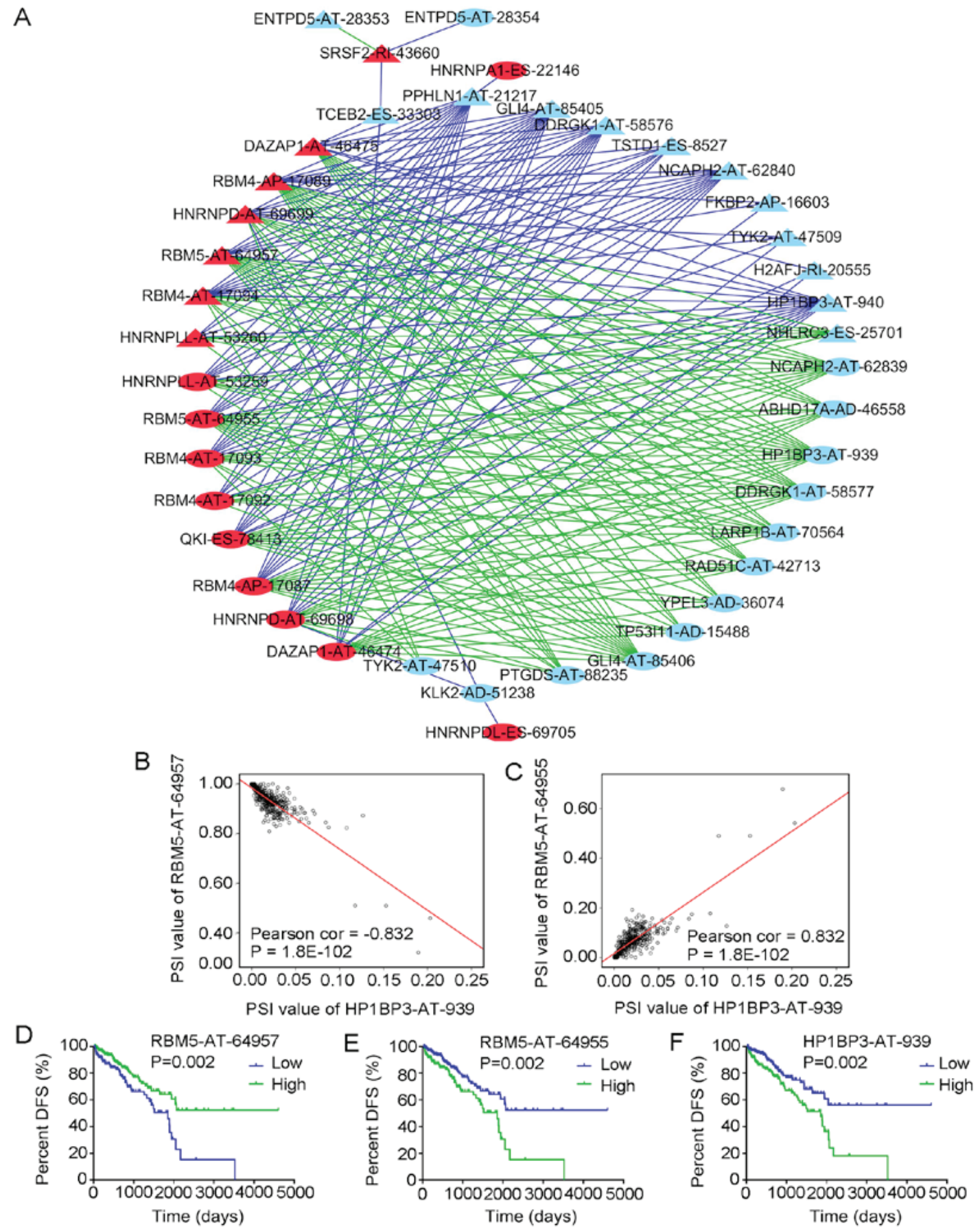

Figure 14. Correlation analysis between splicing events of splicing factors and DFS-associated splicing events. (A) Correlation network of splicing events in PRAD. Red nodes represent splicing events of splicing factors, light blue nodes represent splicing events of non-splicing factors, triangle nodes represent splicing events positively associated with DFS, oval nodes represent splicing events negatively associated with DFS, blue lines represent negative correlations and green lines represent positive correlations. (B) Correlation of the PSI value between RBM5-AT-64957 and HP1BP3-AT-939. RBM5-AT-64957 is one of the splicing events of the splicing factor RBM5. (C) Correlation of the PSI value between RBM5-AT-64955 and HP1BP3-AT-939. RBM5-AT-64955 is one of the splicing events of the splicing factor RBM5. (D-F) Kaplan-Meier curves for evaluating the DFS time of PRAD patients stratified by the median PSI value of (D) RBM5-AT-64957, (E) RBM5-AT-64955 and (F) HP1BP3-AT-939 (high/low). PRAD, prostate adenocarcinoma; DFS, disease-free survival; AT, Alternate Terminator type of splicing events; PSI, percent-spliced-in; RBM4, RNA binding motif protein 4; DAZAP1, DAZ-associated protein 1; QKI, QKI, KH domain containing RNA binding; HNRNPDL, heterogeneous nuclear ribonucleoprotein D like; SRSF2, serine and arginine rich splicing factor 2; TSTD1, thiosulfate sulfurtransferase like domain containing 1; HP1BP3, heterochromatin protein 1 binding protein 3; DDRGK1, DDRGK domain containing 1; GLI4, GLI family zinc finger 4; PPHLN1, periphilin 1; YPEL3, yippee like 3; NCAPH2, non-SMC condensin II complex subunit H2; LARP1B, La ribonucleoprotein domain family member 1B; TYK2, tyrosine kinase 2; ABHD17A, abhydrolase domain containing 17A; H2AFJ, H2A histone family member J; TP53I11, tumor protein p53 inducible protein 11; RAD51C, RAD51 paralog C; KLK2, kallikrein related peptidase 2; PTGDS, prostaglandin D2 synthase; NHLRC3, NHL repeat containing 3; TCEB2, elongin B; FKBP2, FK506 binding protein 2; ENTPD5, ectonucleoside triphosphate diphosphohydrolase 5.

transcripts of genes generated by AS (47-50). In addition, as the major executors of AS, splicing factors have important roles in the production of the splicing variants of genes $(51,52)$.
Therefore, a regulatory network of splicing factors and the AS products of regulated genes was constructed in the present study to elucidate pathogenic mechanisms of PRAD. The 
DFS-SEs identified by univariate Cox analysis were subjected to correlation analyses for the construction of a more accurate correlation network between splicing events of splicing factors and other genes. The splicing events of non-splicing factors associated with a relatively long DFS exhibited a significant negative correlation with the splicing events of the splicing factors, while the opposite trend was identified for splicing events of non-splicing factors associated with poor DFS. At present, no relevant literature is available to confirm the potential regulatory associations for the splicing events discovered in the present study.

The major data of the present study comprehensively assessed the prognostic value of splicing events in PRAD. Of note, the present study had certain limitations. The lack of PRAD-associated mortalities in the TCGA database meant that only 'with tumor' was available as the endpoint in the prognostic analysis, rather than 'death'. Validation in a larger cohort in a future study is also required. The present study also identified numerous splicing events that may theoretically influence the biological behavior of PRAD, so these splicing events require verification in experimental biological studies.

In conclusion, the present study comprehensively assessed AS events in PRAD. PIs generated from DFS-SEs had a high prognostic value. In addition, a more accurate regulatory network for AS in PRAD was provided based on a correlation analysis.

\section{Acknowledgements}

Not applicable.

\section{Funding}

This study was supported by the Innovation Project of Guangxi Graduate Education (grant no. YCBZ2017044), the Promoting Project of Basic Capacity for Young and Middle-aged University Teachers in Guangxi (grant no. KY2016YB090) and the International Communication of Guangxi Medical University Graduate Education (2017) from the Education Department of Guangxi.

\section{Availability of data and materials}

The PSI value of splicing events and clinical information in PRAD analyzed in this study may be acquired from the TCGASpliceSeq and TCGA.

\section{Author's contributions}

ZNM provided the study design. ZGH and RQH performed the analyses and calculations. $\mathrm{ZGH}, \mathrm{RQH}$ and $\mathrm{ZNM}$ all contributed to the writing of the manuscript.

\section{Ethics approval and consent to participate}

Not applicable.

\section{Patient consent for publication}

Not applicable.

\section{Competing interests}

The authors declare that they have no competing interests.

\section{References}

1. Salinas CA, Tsodikov A, Ishak-Howard M and Cooney KA: Prostate cancer in young men: An important clinical entity. Nat Rev Urol 11: 317-323, 2014.

2. Tabayoyong W and Abouassaly R: Prostate Cancer Screening and the Associated Controversy. Surg Clin North Am 95: 1023-1039, 2015.

3. Torre LA, Bray F, Siegel RL, Ferlay J, Lortet-Tieulent J and Jemal A: Global cancer statistics, 2012. CA Cancer J Clin 65: 87-108, 2015.

4. Siegel RL, Miller KD and Jemal A: Cancer statistics, 2018. CA Cancer J Clin 68: 7-30, 2018.

5. Etzioni R, Tsodikov A, Mariotto A, Szabo A, Falcon S, Wegelin J, DiTommaso D, Karnofski K, Gulati R, Penson DF, et al: Quantifying the role of PSA screening in the US prostate cancer mortality decline. Cancer Causes Control 19: 175-181, 2008.

6. Chen W, Zheng R, Baade PD, Zhang S, Zeng H, Bray F, Jemal A Yu XQ and He J: Cancer statistics in China, 2015. CA Cancer J Clin 66: 115-132, 2016.

7. Kontos CK, Adamopoulos PG and Scorilas A: Prognostic and predictive biomarkers in prostate cancer. Expert Rev Mol Diagn 15: 1567-1576, 2015.

8. Dimakakos A, Armakolas A and Koutsilieris M: Novel tools for prostate cancer prognosis, diagnosis, and follow-up. BioMed Res Int 2014: 890697, 2014.

9. Thalgott M, Rack B, Maurer T, Souvatzoglou M, Eiber M, Kress V, Heck MM, Andergassen U, Nawroth R, Gschwend JE, et al: Detection of circulating tumor cells in different stages of prostate cancer. J Cancer Res Clin Oncol 139: 755-763, 2013.

10. Hessels D and Schalken JA: Urinary biomarkers for prostate cancer: A review. Asian J Androl 15: 333-339, 2013.

11. Wagner SD and Berglund JA: Alternative pre-mRNA splicing. Methods Mol Biol 1126: 45-54, 2014.

12. Kelemen O, Convertini P, Zhang Z, Wen Y, Shen M, Falaleeva M and Stamm S: Function of alternative splicing. Gene 514: 1-30, 2013.

13. Krause WC, Shafi AA, Nakka M and Weigel NL: Androgen receptor and its splice variant, AR-V7, differentially regulate FOXA1 sensitive genes in LNCaP prostate cancer cells. Int J Biochem Cell Biol 54: 49-59, 2014.

14. Lapuk AV, Volik SV, Wang Y and Collins CC: The role of mRNA splicing in prostate cancer. Asian J Androl 16: 515-521, 2014.

15. Liu LL, Xie N, Sun S, Plymate S, Mostaghel E and Dong X: Mechanisms of the androgen receptor splicing in prostate cancer cells. Oncogene 33: 3140-3150, 2014.

16. Sprenger CC and Plymate SR: The link between androgen receptor splice variants and castration-resistant prostate cancer. Horm Cancer 5: 207-217, 2014.

17. Omenn GS, Menon R and Zhang Y: Innovations in proteomic profiling of cancers: Alternative splice variants as a new class of cancer biomarker candidates and bridging of proteomics with structural biology. J Proteomics 90: 28-37, 2013.

18. Li Y, Sun N, Lu Z, Sun S, Huang J, Chen Z and He J: Prognostic alternative mRNA splicing signature in non-small cell lung cancer. Cancer Lett 393: 40-51, 2017.

19. Ryan MC, Cleland J, Kim R, Wong WC and Weinstein JN: SpliceSeq: A resource for analysis and visualization of RNA-Seq data on alternative splicing and its functional impacts. Bioinformatics 28: 2385-2387, 2012.

20. Sveen A, Kilpinen S, Ruusulehto A, Lothe RA and Skotheim RI: Aberrant RNA splicing in cancer; expression changes and driver mutations of splicing factor genes. Oncogene 35: 2413-2427, 2016.

21. Yoshida K and Ogawa S: Splicing factor mutations and cancer. Wiley Interdiscip Rev RNA 5: 445-459, 2014.

22. Ogawa S: Splicing factor mutations in myelodysplasia. Int $J$ Hematol 96: 438-442, 2012.

23. Silipo M, Gautrey H and Tyson-Capper A: Deregulation of splicing factors and breast cancer development. J Mol Cell Biol 7: 388-401, 2015.

24. Karni R, de Stanchina E, Lowe SW, Sinha R, Mu D and Krainer AR: The gene encoding the splicing factor SF2/ASF is a proto-oncogene. Nat Struct Mol Biol 14: 185-193, 2007. 
25. Adler AS, McCleland ML, Yee S, Yaylaoglu M, Hussain S, Cosino E, Quinones G, Modrusan Z, Seshagiri S, Torres E, et al: An integrative analysis of colon cancer identifies an essential function for PRPF6 in tumor growth. Genes Dev 28: 1068-1084, 2014.

26. Ryan M, Wong WC, Brown R, Akbani R, Su X, Broom B, Melott J and Weinstein J: TCGASpliceSeq a compendium of alternative mRNA splicing in cancer. Nucleic Acids Res 44: D1018-D1022. 2016.

27. Heagerty PJ, Lumley T and Pepe MS: Time-dependent ROC curves for censored survival data and a diagnostic marker. Biometrics 56: 337-344, 2000.

28. Piva F, Giulietti M, Burini AB and Principato G: SpliceAid 2 A database of human splicing factors expression data and RNA target motifs. Hum Mutat 33: 81-85, 2012

29. Lex A, Gehlenborg N, Strobelt H, Vuillemot R and Pfister H: UpSet: Visualization of intersecting sets. IEEE Trans Vis Comput Graph 20: 1983-1992, 2014.

30. Fredericks AM, Cygan KJ, Brown BA and Fairbrother WG RNA-Binding Proteins: Splicing Factors and Disease. Biomolecules 5: 893-909, 2015.

31. He Y, Lu J, Ye Z, Hao S, Wang L, Kohli M, Tindall DJ, Li B, Zhu R, Wang L, et al: Androgen receptor splice variants bind to constitutively open chromatin and promote abiraterone-resistant growth of prostate cancer. Nucleic Acids Res 46: 1895-1911, 2018.

32. Munkley J, Livermore K, Rajan P and Elliott DJ: RNA splicing and splicing regulator changes in prostate cancer pathology. Hum Genet 136: 1143-1154, 2017.

33. Ciccarese C, Santoni M, Brunelli M, Buti S, Modena A, Nabissi M, Artibani W, Martignoni G, Montironi R, Tortora G, et al: AR-V7 and prostate cancer: The watershed for treatment selection? Cancer Treat Rev 43: 27-35, 2016.

34. Couture F, Sabbagh R, Kwiatkowska A, Desjardins R, Guay SP, Bouchard L and Day R: PACE4 Undergoes an Oncogenic Alternative Splicing Switch in Cancer. Cancer Res 77: 6863-6879, 2017.

35. Hernandez JR, Kim JJ, Verdone JE, Liu X, Torga G, Pienta KJ and Mooney SM: Alternative CD44 splicing identifies epithelia prostate cancer cells from the mesenchymal counterparts. Med Oncol 32: 159, 2015.

36. Cappellari M, Bielli P, Paronetto MP, Ciccosanti F, Fimia GM Saarikettu J, Silvennoinen O and Sette C: The transcriptional co-activator SND1 is a novel regulator of alternative splicing in prostate cancer cells. Oncogene 33: 3794-3802, 2014.

37. Robert $\mathrm{C}$ and Watson $\mathrm{M}$ : The incredible complexity of RNA splicing. Genome Biol 17: 265, 2016

38. Brasky TM, Darke AK, Song X, Tangen CM, Goodman PJ, Thompson IM, Meyskens FL Jr, Goodman GE, Minasian LM, Parnes HL, et al: Plasma phospholipid fatty acids and prostate cancer risk in the SELECT trial. J Natl Cancer Inst 105: $1132-1141,2013$

39. Bassett JK, Severi G, Hodge AM, MacInnis RJ, Gibson RA, Hopper JL, English DR and Giles GG: Plasma phospholipid fatty acids, dietary fatty acids and prostate cancer risk. Int J Cancer 133: 1882-1891, 2013.
40. Moreel X, Allaire J, Léger C, Caron A, Labonté MÈ, Lamarche B, Julien P, Desmeules P, Têtu B and Fradet V: Prostatic and dietary omega-3 fatty acids and prostate cancer progression during active surveillance. Cancer Prev Res (Phila) 7: 766-776, 2014.

41. Gang X, Yang Y, Zhong J, Jiang K, Pan Y, Karnes RJ, Zhang J, $\mathrm{Xu} \mathrm{W}$, Wang $\mathrm{G}$ and Huang $\mathrm{H}$ : P300 acetyltransferase regulates fatty acid synthase expression, lipid metabolism and prostate cancer growth. Oncotarget 7: 15135-15149, 2016

42. Xiao D, Powolny AA, Moura MB, Kelley EE, Bommareddy A, Kim SH, Hahm ER, Normolle D, Van Houten B and Singh SV: Phenethyl isothiocyanate inhibits oxidative phosphorylation to trigger reactive oxygen species-mediated death of human prostate cancer cells. J Biol Chem 285: 26558-26569, 2010.

43. Chen ZP, Li M, Zhang LJ, He JY, Wu L, Xiao YY, Duan JA, Cai T and Li WD: Mitochondria-targeted drug delivery system for cancer treatment. J Drug Target 24: 492-502, 2016.

44. Goltz D, Gevensleben H, Dietrich J, Ellinger J, Landsberg J, Kristiansen G and Dietrich D: Promoter methylation of the immune checkpoint receptor PD-1 (PDCD1) is an independent prognostic biomarker for biochemical recurrence-free survival in prostate cancer patients following radical prostatectomy. OncoImmunology 5: e1221555, 2016.

45. Holmes EE, Goltz D, Sailer V, Jung M, Meller S, Uhl B, Dietrich J, Röhler M, Ellinger J, Kristiansen G, et al: PITX3 promoter methylation is a prognostic biomarker for biochemical recurrence-free survival in prostate cancer patients after radical prostatectomy. Clin Epigenetics 8: 104, 2016.

46. Meller S, Zipfel L, Gevensleben H, Dietrich J, Ellinger J, Majores M, Stein J, Sailer V, Jung M, Kristiansen G, et al: CDO1 promoter methylation is associated with gene silencing and is a prognostic biomarker for biochemical recurrence-free survival in prostate cancer patients. Epigenetics 11: 871-880, 2016.

47. Gonzàlez-Porta M, Frankish A, Rung J, Harrow J and Brazma A Transcriptome analysis of human tissues and cell lines reveals one dominant transcript per gene. Genome Biol 14: R70, 2013.

48. Soussi T, Leroy B and Taschner PE: Recommendations for analyzing and reporting TP53 gene variants in the highthroughput sequencing era. Hum Mutat 35: 766-778, 2014.

49. Thadani-Mulero M, Portella L, Sun S, Sung M, Matov A, Vessella RL, Corey E, Nanus DM, Plymate SR and Giannakakou P: Androgen receptor splice variants determine taxane sensitivity in prostate cancer. Cancer Res 74: 2270-2282, 2014.

50. Carstens RP, Eaton JV, Krigman HR, Walther PJ and GarciaBlanco MA: Alternative splicing of fibroblast growth factor receptor 2 (FGF-R2) in human prostate cancer. Oncogene 15: 3059-3065, 1997.

51. Anczuków O and Krainer AR: Splicing-factor alterations in cancers. RNA 22: 1285-1301, 2016.

52. Lee Y and Rio DC: Mechanisms and regulation of alternative Pre-mRNA splicing. Annu Rev Biochem 84: 291-323, 2015.

(i) $\Theta$ This work is licensed under a Creative Commons Attribution-NonCommercial-NoDerivatives 4.0 International (CC BY-NC-ND 4.0) License. 